\title{
BMSAP
}

Bulletins et mémoires de la Société d'Anthropologie de Paris

BMSAP

$33(1) \mid 2021$

Les 1000 premiers jours de vie dans les populations du présent et du passé

\section{Asymétries directionnelles et fluctuantes en période périnatale : étude exploratoire dans les populations du passé}

Directional and fluctuating asymmetries in perinates: An exploratory study on past populations

Caroline Partiot, Dominique Castex, Mark Guillon, Camille Noûs, Bruno Maureille et Frédéric Santos

\section{(2) OpenEdition}

Journals

Édition électronique

URL : https://journals.openedition.org/bmsap/7476

DOI : $10.4000 /$ bmsap.7476

ISSN : $1777-5469$

Éditeur

Société d'Anthropologie de Paris

\section{Référence électronique}

Caroline Partiot, Dominique Castex, Mark Guillon, Camille Noûs, Bruno Maureille et Frédéric Santos, «Asymétries directionnelles et fluctuantes en période périnatale : étude exploratoire dans les populations du passé », Bulletins et mémoires de la Société d'Anthropologie de Paris [En ligne], 33 (1) | 2021, mis en ligne le 27 avril 2021, consulté le 18 mars 2022. URL : http://journals.openedition.org/ bmsap/7476 ; DOI : https://doi.org/10.4000/bmsap.7476

Les contenus des Bulletins et mémoires de la Société d'Anthropologie de Paris sont mis à disposition selon les termes de la licence Creative Commons Attribution-NonCommercial-NoDerivatives 4.0 International License. 


\title{
Asymétries directionnelles et fluctuantes en période périnatale : étude exploratoire dans les populations du passé
}

\author{
Directional and fluctuating asymmetries in perinates: \\ An exploratory study on past populations
}

\author{
Caroline Partiot (D) ${ }^{1,2 *}$, Dominique Castex ${ }^{3}$, Mark Guillon ${ }^{3,4}$, Camille Noûs ${ }^{5}$, Bruno Maureille (D) ${ }^{3}$, Frédéric Santos (D) ${ }^{3}$ \\ 1 UMR 7206 Eco-Anthropologie (EA), MNHN, CNRS, Musée de l'Homme, Université de Paris Diderot, Paris, France \\ 2 Espace muséographique et centre d'interprétation du Paléosite, Saint-Césaire, France \\ 3 UMR 5199 PACEA, Univ. Bordeaux, CNRS, MCC, Pessac, France \\ 4 INRAP, Le Grand Quevilly, France \\ 5 Laboratoire Cogitamus, Université de Bordeaux, Pessac, France \\ * caroline.partiot@mnhn.fr
}

Reçu : 27 avril 2020 ; accepté : 10 juillet 2020

Bulletins et Mémoires de la Société d'Anthropologie de Paris

Cette note fait suite à une communication présentée lors des $1845^{\text {es }}$ journées de la Société d'Anthropologie de Paris dans le cadre de la session "Les 1000 premiers jours de vie dans les populations du présent et du passé"

Résumé - Les asymétries directionnelles (expression préférentielle d'un caractère d'un côté plutôt que de l'autre) et les asymétries fluctuantes (écarts mineurs, aléatoires et indépendants par rapport à la symétrie bilatérale) font l'objet d'un nombre croissant d'analyses dans des corpus actuels de sujets décédés en période périnatale, du fait de leurs implications respectives dans les questions de latéralisation préférentielle et de stabilité du développement. Ces axes de recherche sont toutefois rarement investis dans cette classe d'âge et dans les populations du passé. Notre étude s'est ainsi donné pour objectif de rechercher la présence ou l'absence de ces deux types d'asymétries dans un corpus archéologique, via une approche exploratoire adaptant les protocoles existants aux spécificités de ce matériel ostéologique. Réalisée sur un corpus de 116 individus issus de trois collections (nécropole 8B-51, île de Saï, Soudan, Kerma classique ; cimetière de Blandy-les-Tours, Bassin parisien, $\mathrm{X}^{\mathrm{e}}-\mathrm{XII}{ }^{\mathrm{e}}$ siècles ; cimetière de Provins, Bassin parisien, $\mathrm{XIII}^{\mathrm{e}}$ XVIII ${ }^{\mathrm{e}}$ siècles), cette étude apporte des éléments de réflexion quant à la faisabilité méthodologique de ces analyses sur os sec, en permettant d'identifier des variables exprimant ces asymétries. Ces résultats préliminaires visent à engager la discussion quant à l'emploi de ces marqueurs biologiques pour les analyses ostéobiographiques de sujets immatures issus de populations du passé, notamment en tant qu'estimateur des perturbations du développement.

Mots clés - symétrie, latéralisation préférentielle, instabilité du développement, stress, décès périnatal, fœus

Abstract - Directional asymmetry (the preferential expression of a character on one side rather than the other) and fluctuating asymmetry (minor, random and independent deviations from bilateral symmetry) are increasingly investigated in modern corpuses of deceased perinates, since they are involved in assessing preferential lateralization and developmental stability, respectively. However, few studies have examined these issues in perinates from past populations. Our study was conducted to investigate the absence or presence of these two types of asymmetry in an archaeological corpus, using an exploratory approach with a view to adapting existing protocols to the specific features of this osteological material. The study was carried out on a corpus of 116 individuals from three collections (Necropolis 8B-51 from Saï Island, Sudan, Classic Kerma period, the Blandy-les-Tours cemetery in the Paris Basin, $10^{\text {th }}$ $13^{\text {th }}$ centuries, and the Provins cemetery in the Paris Basin, $12^{\text {th }}-18^{\text {th }}$ centuries). The investigation produced an assessment of the methodological feasibility of these analyses for skeletonized remains and allowed to identify several variables expressing their asymmetries. We expect these preliminary results to initiate discussions on the use of this type of biological for osteobiographical analyses of immature individuals from past populations, and particularly as an estimator of developmental disturbances.

Keywords - symmetry, handedness, developmental instability, stress, perinatal death, foetuses

\section{Introduction}

La symétrie, définie comme la similitude exacte de deux éléments par rapport à un point, un axe ou un plan de référence, de même que l'asymétrie, ou déviation par rapport 
à cette symétrie, comptent parmi les principes théoriques fondamentaux des processus de développement (e.g. Cooke, 2004). Deux types d'asymétries sont classiquement distingués dans la littérature scientifique. Le premier est désigné sous l'appellation d'asymétries directionnelles (AD) (Van Valen, 1962). Cette expression préférentielle d'un caractère d'un côté plutôt que de l'autre se traduit graphiquement par une distribution normale des écarts entre le côté droit et le côté gauche, avec une moyenne des écarts différente de zéro (Van Valen, 1962). La présence d'AD au niveau squelettique a été très tôt recherchée chez l'homme, et en particulier au tout début de la vie, comme une possible expression de l'origine génétique de la latéralisation préférentielle du comportement, voire de la préférence manuelle (Schultz, 1923 ; Steele et Mays, 1995 ; Gawlikowska et al., 2007 ; Van Dongen et al., 2010).

Le second type d'asymétries usuellement étudié est celui des asymétries fluctuantes (AF), définies comme des écarts mineurs, aléatoires et indépendants par rapport à la symétrie bilatérale. Elles se traduisent graphiquement par une distribution normale des écarts entre le côté droit et le côté gauche, avec une moyenne des écarts égale à zéro (Van Valen, 1962). L'interprétation biologique des AF est fondée sur le principe selon lequel des traits bilatéraux se développant sous l'action du même génome et dans le même environnement doivent, en théorie, présenter une symétrie. En pratique, l'organisme doit amortir l'impact de micro-perturbations du développement, dont les effets sont cumulatifs et aléatoires, avec des capacités d'intégration elles-mêmes variables selon les individus ou le trait considéré (Klingenberg, 2003). La présence de ces asymétries fluctuantes constituerait ainsi le signal d'une instabilité du développement, avec des écarts ayant tendance à augmenter au fur et à mesure que décroîtrait la propension de l'organisme à amortir l'impact de ces facteurs de stress environnementaux (Livshits et al., 1988 ; Palmer et Strobeck, 1992 ; Van Dongen, 2006). Selon ce principe, les asymétries fluctuantes apparaissent comme un indicateur du degré d'instabilité du développement d'une population (Palmer, 1994 ; Klingenberg, 2003 ; Van Dongen, 2006; Van Dongen et al., 2010 ; Van Dongen et Gangestad, 2011).

Les variables identifiées comme exprimant ces asymétries, appelées "variables-support", sont principalement acquises sur des sujets adultes, et concernent majoritairement les tissus mous (Livshits et al., 1988 ; Livshits et Kobyliansky, 1989 ; Kujanová et al., 2008 ; Özener et Graham, 2014). S’il existe des données sur l'expression osseuse de ces asymétries au tout début de la vie humaine (Van Dongen et al., 2010 ; Bots et al., 2015), elles restent limitées à des corpus actuels, avec un faible nombre de dimensions étudiées (moins de dix variables), et touchent uniquement aux longueurs maximales des os longs du squelette appendiculaire (Steele et Mays, 1995 ; Van Dongen et al., 2010). Les études les plus récentes sont, par ailleurs, souvent réalisées à partir de radiographies, avec des protocoles adaptés aux biais relatifs à ce type de support. Les analyses réalisées sur des populations du passé demeurent rares (Steele et Mays, 1995 ; Storm, 2010). L'étude de Steele et Mays (1995) porte ainsi sur des sujets décédés en période périnatale issus d'une collection archéologique, et identifie de l'AD au niveau des longueurs maximales des os du membre supérieur uniquement, et ce dans un corpus de dimensions très réduites (14 individus). Les travaux de Storm (2010) portent sur davantage d'individus et un grand nombre de variables métriques, mais analysent les $\mathrm{AF}$ et $\mathrm{AD}$ dans un corpus constitué de sujets adultes et de sujets non-adultes, sans distinction entre les classes d'âge immatures. Les asymétries squelettiques des sujets décédés dans la période périnatale issus des populations du passé ne sont, de fait, presque pas documentées, alors même que les dynamiques actuelles poussent à la mise à disposition croissante de matériel, et à un intérêt renouvelé pour l'analyse ostéobiographique des sujets immatures.

Notre étude s'est donné pour objectif d'apporter des éléments de réponse à la question de la présence ou de l'absence d'AD et d'AF dans un large corpus archéologique constitué de sujets décédés dans la période périnatale comprise au sens large - entre 22 semaines d'aménorrhée et 2 mois post-partum (Partiot, 2018 ; 2020). Cette réflexion méthodologique a eu pour objectifs de (i) mettre en place une approche exploratoire visant à adapter les protocoles existants aux spécificités des collections archéologiques ; (ii) détecter la présence d'AD afin de questionner la possible expression osseuse d'une latéralisation préférentielle dès le stade périnatal ; (iii) étudier la présence d'AF afin d'interroger la pertinence de ce marqueur pour l'identification des perturbations du développement subies par une population.

\section{Matériel}

Le corpus d'étude était constitué de 116 individus issus de trois collections ostéologiques (Partiot, 2018). La première comporte 54 individus soudanais datés du Kerma classique (nécropole 8B-51 de l'île de Saï, XVe-XIV siècles av. J.-C.) (Murail et al., 2004 ; Maureille et al., 2006), la deuxième 27 sujets du cimetière de l'église dite anonyme de Blandyles-Tours ( $\mathrm{X}^{\mathrm{e}}-\mathrm{XII}{ }^{\mathrm{e}}$ siècles ap. J.-C.) (Delattre, 2008) et la troisième 35 sujets de l'église et du cimetière paroissial de Provins (XIII'-XVIII ${ }^{\mathrm{e}}$ siècles ap. J.-C.) (Guillon et al., 2002 ; Portat, 2018). L'âge au décès des individus a été estimé en première intention en semaines d'aménorrhée sur la base des longueurs diaphysaires maximales grâce aux formules de Fazekas et Kósa (1978) révisées par Sellier (dans Schmitt et Georges, 2008). La méthode a été privilégiée car elle est applicable à sept os longs différents et a été testée sur des populations de référence, sur des sujets âgés du stade fœtal jusqu'à 4 ans (Sellier et al., 1997). La fiabilité des résultats obtenus par l'intermédiaire d'une estimation staturale apparait comme assurée dans cette classe d'âge du fait des très fortes dynamiques de croissance longitudinale. Les germes dentaires étaient par ailleurs insuffisamment conservés pour que l'estimation de l'âge au décès puisse être basée sur leur minéralisation. Le décompte des incréments dentaires et l'observation histologique de la ligne néonatale n'ont, de même, pas été réalisés, l'histologie classique n'étant pas 
privilégiée car destructive, et les méthodes d'histologie virtuelle étant financièrement et matériellement inaccessibles.

En seconde intention, pour les individus dont la conservation osseuse était insuffisante, l'âge au décès a été approché par comparaison de format, avec seulement pour objectif d'écarter les sujets dont l'âge au décès était supérieur à 48 semaines d'aménorrhée. Les estimations obtenues s'échelonnent ainsi entre 24 et 47 semaines d'aménorrhée, avec 19 sujets décédés entre 24 et 34 semaines d'aménorrhée révolues, 60 décédés entre 35 et 40 semaines d'aménorrhée révolues, et 37 décédés entre 41 et 47 semaines d'aménorrhée révolues.

Dans la mesure où il s'agit d'individus non-survivants, la question de la nature plus ou moins pathologique du corpus, et donc de la représentativité des résultats par rapport à ceux qui devraient être obtenus sur une population naturelle, se pose néanmoins, de même que la place accordée au paradoxe ostéologique dans cette classe d'âge (Wood et al., 1992). D'une manière générale, plusieurs éléments peuvent amener à envisager que ce type de corpus ne serait pas systématiquement à considérer comme étant de nature fortement pathologique. Le premier est qu'une grande partie des décès périnataux survenant actuellement dans des contextes faiblement médicalisés (les plus à mêmes de se rapprocher des contextes connus par les populations pré-jennériennes) est rapportée comme se produisant juste après la naissance - $70 \%$ d'entre eux surviennent aujourd'hui dans les trois premiers jours de vie postnatale (Engmann et al., 2011 ; Séguy, 2018) - et sont dus à des circonstances sans rapport avec un développement anormal du fœtus. Les principales causes de ces décès des premiers jours sont en effet liées à des accidents de la naissance (asphyxie, septicémie, hémorragie, accouchement traumatique et/ou prolongé) ou à des conséquences de la prématurité (vulnérabilité à l'hypothermie) (Séguy, 2018). Les décès plus précoces (décès intra-utérins ou mortnés) peuvent pour leur part être dus à des pathologies maternelles, tandis qu'une grande partie des décès survenant dans les semaines qui suivent est également en rapport avec la malnutrition (difficultés d'allaitement), le tétanos néonatal, le comportement plus ou moins attentif des proches, ou encore les pathologies transmises par ces derniers (troubles gastro-intestinaux, virus grippaux, etc.) et contre lesquelles le système immunitaire fortement immature des nourrissons peine à réagir (Zupan et Åhman, 2006). Même si cette fréquence a tendance à varier en fonction du contexte socioéconomique, on estime ainsi que $1 \%$ des nouveau-nés sont aujourd'hui porteurs d'anomalies congénitales majeures (Zupan et Åhman, 2006), un grand nombre des sujets atteints de troubles du développement faisant aussi l'objet de fausses couches naturelles précoces. On peut donc avancer qu'une proportion, certes difficile à évaluer, des sujets décédés au cours de la période périnatale dans les populations anciennes ne sont pas porteurs d'anomalies majeures du développement ou de pathologies graves, et ne seraient pas à même d'être considérés comme "hors-normes". Plus spécifiquement, l'existence d'anomalies du développement a toutefois pu être discutée pour 27 individus appartenant à la collection de l'île de Saï, et ce en raison de la présence sur leur squelette de côtes surnuméraires cervicales, caractéristique anatomique associée à des mutations dans les gènes Hox et identifiée comme un critère de morbidité pour la classe d'âge périnatale (Partiot et al., 2020). Il pourrait cependant s'agir d'un cas relativement particulier, puisque des facteurs comme la nature insulaire de la population, l'existence de proximités génétiques, voire de phénomènes de consanguinité, auraient pu contribuer à augmenter le taux d'anomalies du développement (Partiot, 2018 ; Partiot et al., 2020). L'étude biologique de ces individus, tout comme celle des autres individus du corpus, n'a cependant révélé aucune anomalie ou pathologie osseuse "manifeste" (Partiot, 2018).

La question de la représentativité des résultats obtenus sur ce type de corpus ostéologique doit toutefois être maintenue au premier plan des perspectives de cet axe de recherche, même si ce point ne va pas sans soulever un certain nombre de difficultés. Il n'est en effet évidemment pas envisageable, pour des raisons éthiques et techniques, de réaliser des analyses prospectives sur des corpus proches d'une population naturelle (par exemple, lors des examens obstétriques de contrôle), et les études actuelles sont elles-mêmes majoritairement réalisées sur des collections issues de contexte médico-légal, et donc majoritairement non représentatives d'une population naturelle. Même s'il apparaît qu'il s'agit d'un biais inhérent aux analyses réalisées sur des sujets appartenant à la classe d'âge périnatale, il est donc à prévoir que seul le développement de cet axe de recherche et l'analyse de la variabilité inter-populationnelle permettront à l'avenir de préciser si les niveaux d'asymétries identifiés sont typiques de la classe d'âge, ou reflètent l'état de morbidité des sujets.

\section{Méthodes}

Le protocole métrique considéré en première intention comprenait 197 variables métriques linéaires issues en grande majorité des publications de références de Fazekas et Kósa (1978), Schutkowski (1990; 1993) et Duday et al. (1995). Elles font l'objet d'une synthèse descriptive dans Partiot (2018). Elles ont été acquises au pied à coulisse électronique avec une précision au $10^{\mathrm{e}}$ de millimètre. Parmi ces 197 variables, 106, acquises sur le bloc crânio-facial, le membre supérieur, le membre inférieur et les ceintures, ont été sélectionnées pour les analyses d'asymétries. Les variables acquises sur le gril costal, le rachis, les métacarpiens, métatarsiens et phalanges n'ont pas été prises en compte en raison de leur faible représentation.

\section{Résultats}

\section{Mise en place du protocole exploratoire}

Le choix du protocole comptant parmi les enjeux principaux des études d'asymétries, la multiplication des méthodes entre les différentes études apparaît comme un obstacle majeur aux approches comparatives. Palmer et Strobeck (1986) 
dénombrent ainsi pas moins de 22 méthodes publiées dans la littérature scientifique, lesquelles se distinguent notamment par le mode de prise en compte de l'erreur de mesure (EM), biais fondamental de ce type d'analyses. L'EM est en effet systématiquement comprise dans le calcul de l'écart entre le côté gauche et le côté droit, et peut être du même ordre de grandeur que l'AF et l'AD. Notre revue bibliographique a mis en évidence que deux protocoles, ceux de Palmer et Strobeck (1986) et de Van Dongen et al. (1999), comptent parmi les plus couramment employés et sont utilisés dans les études portant sur les corpus de fotus actuels.

Palmer et Strobeck (1986) définissent différents indicateurs permettant de quantifier l'AF et l'AD de manière aisément interprétable. Pour tester la significativité de ces deux composantes de l'asymétrie en aval de leur quantification, nous avons utilisé le cadre statistique proposé par Van Dongen et al. (1999), affinant le modèle original de Palmer et Strobeck (1986). Un obstacle notable s'est toutefois distingué dans le contexte de l'application de ces protocoles à un corpus issu du registre archéologique. Pour la prise en compte de l'EM, les deux variantes (ANOVA mixte à deux facteurs pour Palmer et Strobeck 1986, modèle de régression à effets mixtes pour Van Dongen et al. 1986), nécessitaient l'acquisition d'une seconde série de mesures pour les côtés gauche et droit. Cette étape constitue, de par son caractère chronophage, un inconvénient notable dans le cadre d'une étude intégrant de nombreuses variables.

Nous avons proposé une adaptation des protocoles avec deux étapes :

Étape 1 : exclusion des variables ne remplissant pas les critères préalables à une identification en tant que variablessupport potentielles, c'est-à-dire, parmi les 106 variables linéaires présélectionnées, exclusion de celles qui ne sont pas susceptibles d'exprimer des AD et AF statistiquement significatives. Cette première étape comportait plusieurs phases successives :

- E1-0 : exclusion des variables insuffisamment répétables, c'est-à-dire celles dont les résultats aux tests intraobservateurs n'étaient pas considérés comme suffisants (coefficient de concordance de Lin (1989) inférieur à $0,95)$;

- E1-1 : exclusion des variables ne respectant pas le prérequis d'indépendance entre l'asymétrie et l'ordre de grandeur de la mesure, effectué par l'application d'un test de corrélation de Pearson entre l'amplitude des erreurs de mesure et la variable considérée, le test étant considéré comme significatif en cas de p-valeur inférieure à 0,05 (Palmer et Strobeck, $1986 ; 1992$ );

- E1-2 : sélection des variables exprimant potentiellement de l'AD via la recherche d'un biais systématique dans une direction, par l'application d'un test de Wilcoxon pour données appariées (considéré comme significatif en cas de p-valeur inférieure à 0,05 ). L'asymétrie directionnelle moyenne a été calculée en millimètres et en pourcentages, pour chaque variable identifiée comme significative ;
- E1-3 : pour chacune des variables non identifiées comme significatives à la présence d'AD, a été quantifiée la présence d'AF par l'application du protocole de Palmer et Strobeck (1986). L'indice no $2 \sum\left[\left(\mathrm{A}_{\mathrm{i}}\right) /\left(\left(\mathrm{D}_{\mathrm{i}}+\mathrm{G}_{\mathrm{i}}\right) / 2\right)\right]$ / $\mathrm{N}^{1}$ a permis d'obtenir pour chaque variable le pourcentage moyen d'AF dans l'échantillon. L'indice no 1 $\sum\left(\mathrm{A}_{\mathrm{i}}\right) / \mathrm{N}^{1}$ a ensuite permis d'obtenir pour chaque variable la moyenne des valeurs absolues des AF sur tout l'échantillon ;

- E1-4 : exclusion des variables pour lesquelles l'EM moyenne apparaissait comme supérieure ou égale aux $\mathrm{AD}$ et $\mathrm{AF}$ identifiées, après calcul de la valeur absolue de l'erreur de mesure moyenne estimée en millimètres et en pourcentages à partir des séries de données acquises pour réaliser les tests intra-observateurs. Par exemple, la variable Il5 a été identifiée comme présentant une AF de l'ordre de $1 \mathrm{~mm}$, soit 10,6 \% de la variable, au cours de l'étape E1-3. L'erreur de mesure moyenne pour cette variable est de $0,6 \mathrm{~mm}$, soit $0,9 \%$ de la variable, ce qui apparaît comme négligeable en regard de l'estimation de l'AF. La variable I15 a donc été conservée parmi les variables pouvant potentiellement exprimer une AF significative.

Les 61 variables n'ayant pas été exclues à l'issue de cette première phase ont été considérées comme des variablessupport potentielles, pour lesquelles une nouvelle acquisition de données sur le matériel a été ensuite réalisée.

Étape 2 : identification et quantification de l'AD et de l'AF par application conjointe des protocoles de Palmer et Strobeck (1986) et de Van Dongen et al. (1999) :

- E2-0 : étape de prérequis : vérification de la normalité des données par un test de normalité de Shapiro-Wilk, et exclusion des variables ne respectant pas le prérequis d'indépendance entre l'asymétrie et l'ordre de grandeur de la mesure, effectué par l'application d'un test de corrélation de Pearson entre l'amplitude des erreurs de mesure et la variable considérée, le test étant considéré comme significatif en cas de p-valeur inférieure à 0,05 (Palmer et Strobeck, 1986 ; 1992);

- E2-1 : évaluation de la significativité de la présence d'AD et d'AF par application du protocole de Van Dongen et al. (1999). Cette évaluation passe par l'estimation du modèle de régression mixte par vraisemblance restreinte REML ( $c f$. Annexe 1);

- E2-2 : estimation quantitative d'AD et d'AF par application du protocole de Palmer et Strobeck (1986) avec les indices utilisés au cours de la phase E1-3, sur les variables identifiées comme présentant une asymétrie statistiquement significative durant la phase E2-1.

1 Avec $\mathrm{A}_{\mathrm{i}}=$ l'asymétrie pour une variable donnée et un individu donné, $\mathrm{R}_{\mathrm{i}}=$ dimension du côté droit, $\mathrm{L}_{\mathrm{i}}=$ dimension du côté gauche, $\mathrm{N}=$ nombre d'individus dans l'échantillon. 
Résultats des analyses d'asymétries directionnelles et d'asymétries fluctuantes

D'après les résultats du protocole exploratoire mis en place, les variables-support exprimant une AD statistiquement significative dans le corpus archéologique étaient au nombre de cinq et étaient, comme attendu, de très faible ampleur, entre 0,1 et 0,3 $\mathrm{mm}$ en moyenne ( $c f$. Annexe 2). Quatre d'entre elles touchaient le membre supérieur et sa ceinture, et une le membre inférieur (figure 1A). À l'exception de la variable Il7 (diamètre antéro-postérieur maximal de l'extrémité distale de la fibula), toutes les dimensions apparaissaient comme étant plus grandes du côté gauche du squelette.

Les variables-support exprimant une AF statistiquement significative dans le corpus archéologique étaient au nombre de 28 et touchaient majoritairement au membre supérieur ainsi qu'aux ceintures scapulaire et pelvienne (figure 1B ; Annexe 3). Les AF identifiées variaient entre 1 et $7 \%$ des variables, avec des écarts $>0,5 \mathrm{~mm}$. Elles concernaient majoritairement le membre supérieur et sa ceinture, de même que la ceinture du membre inférieur.
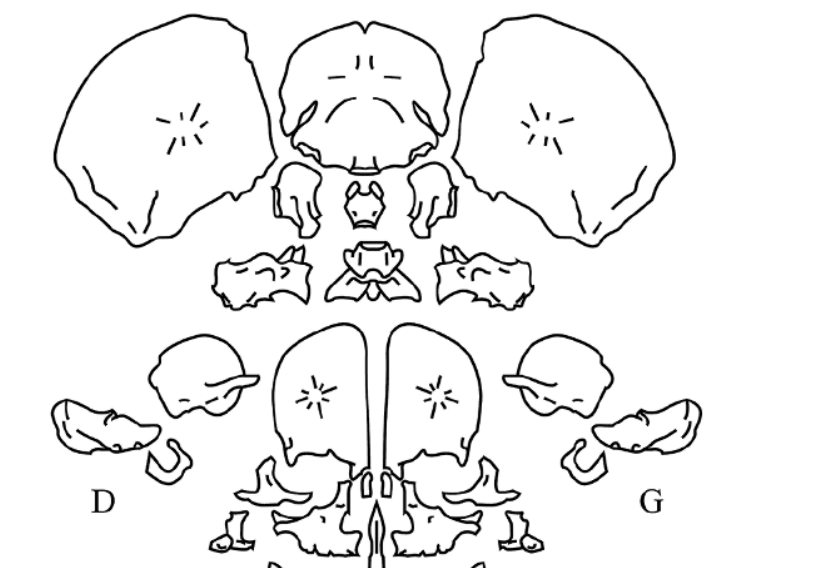

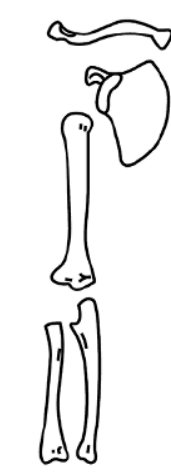

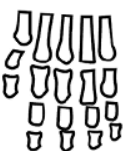
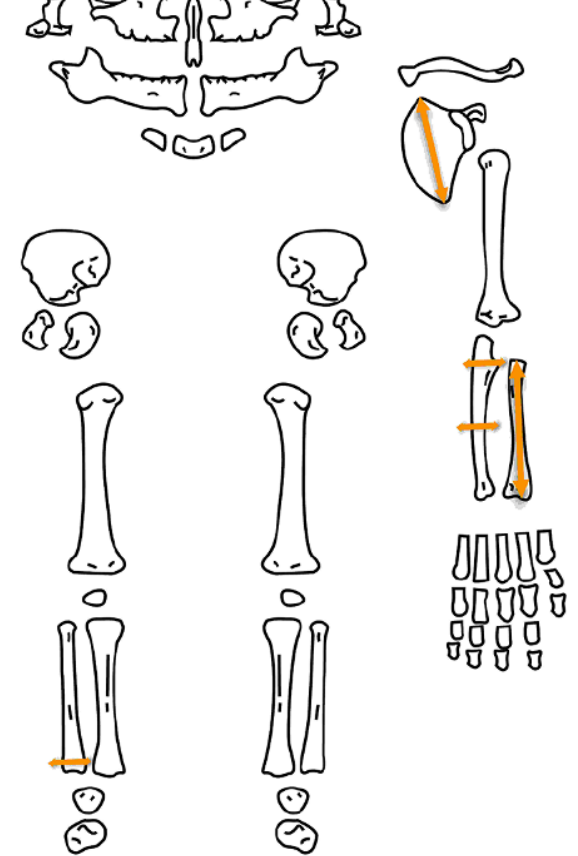

A
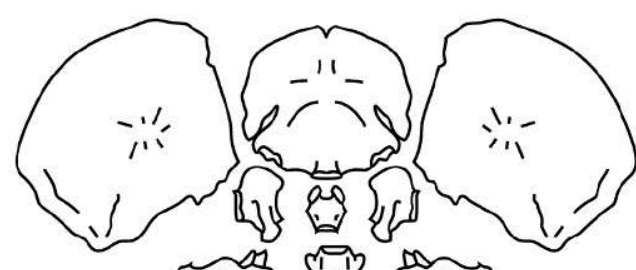

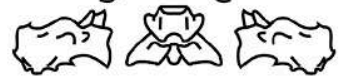

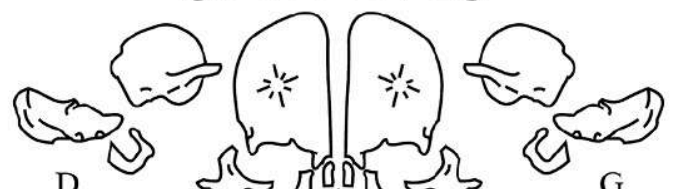

$\mathrm{D}$

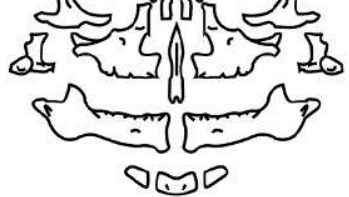

G
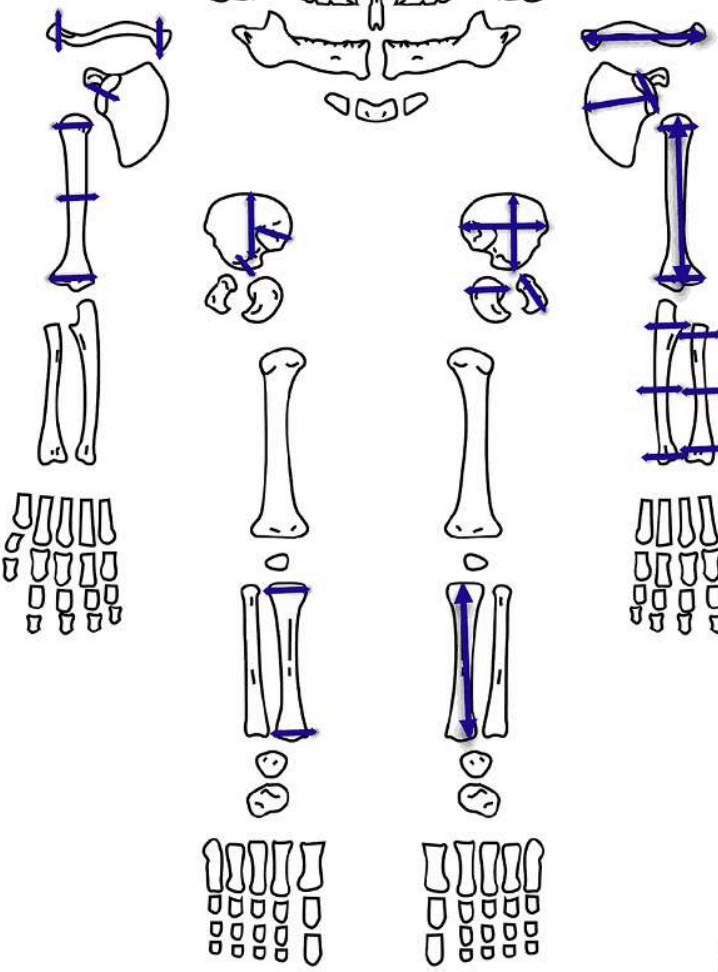

B

Figure 1. Localisation anatomique des variables identifiées comme exprimant des asymétries statistiquement significatives dans le corpus archéologique. (a) asymétrie directionnelle. (b) asymétrie fluctuante, avec D : côté droit ; G : côté gauche. Figure réalisée d'après la fiche de conservation pour sujets décédés en période périnatale, UMR 5809 CNRS, T.S. ConstandseWestermann et C. Meikeljohn, modifiée par A.M. Tillier et H. Duday, DAO : M. Coutureau (Inrap) / Anatomical location of the variables for which statistically significant asymmetries were identified in the archaeological corpus. (a) directional asymmetry. (b) fluctuating asymmetry (D: right side; G: left side). Figure based on the conservation form for perinates, UMR 5809 CNRS, T.S. Constandse-Westermann and C. Meikeljohn, modified by A.M. Tillier and H. Duday, CAD: M. Coutureau (Inrap) 


\section{Discussion et perspectives}

\section{Intérêt de l'approche méthodologique exploratoire}

Du point de vue méthodologique, cette approche exploratoire a eu pour intérêt premier de proposer une adaptation des protocoles existants aux spécificités des corpus issus de collections archéologiques. En étant applicable sur os sec et sur de nombreuses variables linéaires acquises au pied à coulisse, elle a eu pour objectif de favoriser les approches comparatives, en facilitant les analyses portant sur d'autres corpus issus de populations du passé. Le protocole a permis de mettre en évidence un grand nombre de variables-support, ce qui est également un atout, considérant le nombre réduit de variables identifiées dans les études réalisées sur des corpus actuels. Nos résultats ont eu également pour intérêt d'identifier la présence d'AD et d'AF au niveau de dimensions différentes de celles habituellement exploitées dans les études portant sur des corpus actuels, qui sont majoritairement des longueurs maximales des os longs du squelette appendiculaire (Van Dongen et al., 2010). Les variables-support identifiées dans ce corpus archéologique étaient nombreuses à concerner les régions métaphysaires des os longs ou les ceintures pelvienne et scapulaire.

\section{Interprétation biologique des résultats sur l'AD}

La question de l'origine, développementale ou mécanique, de la présence d'AD, a fait l'objet de nombreux débats depuis les premières études anthropologiques sur le développement humain (Fishel, 1929, cité par Fazekas et Kósa, 1978). La latéralisation comportementale (incluant la préférence manuelle) étant reconnue comme liée à la latéralisation génétique du système neurologique, l'hypothèse a été avancée selon laquelle l'AD pourrait être présente au niveau des longueurs osseuses dès la période fœtale et périnatale, comme une préadaptation à l'utilisation prédominante d'un côté du squelette. Chez les humains, cette latéralisation préférentielle correspond à l'utilisation majoritaire du membre supérieur du côté droit (Van Dongen et al., 2010). Des phénomènes d'asymétries croisées, soit l'utilisation préférentielle et compensatoire du membre inférieur du côté opposé au membre supérieur, sont également observés (Auerbach et Ruff, 2006 ; Storm, 2010). À l'inverse, selon l'hypothèse selon laquelle l'AD se développerait au fur et à mesure des sollicitations mécaniques asymétriques et en fonction de facteurs environnementaux au cours de la vie de l'individu, elle devrait en revanche être absente dans les classes d'âge infantiles (e.g. Tang et Verstynen, 2002 ; Van Dongen et al., 2010). À cet égard, l'étude ancienne de Fishel (1929, citée par Fazekas et Kósa, 1978) identifie une dissymétrie générale en faveur du côté droit, résultats que l'on retrouve dans des travaux plus récents, comme ceux d'Adalian (2001), qui identifie des paramètres métriques présentant des moyennes légèrement supérieures du côté droit. Ces observations ne sont en revanche pas confirmées par nos résultats, comme c'est le cas dans les études réalisées avec les protocoles dérivés de Palmer et Strobeck (1986). D'après nos analyses, peu de variables exprimaient une AD significative dans la classe d'âge périnatale, observations qui vont dans le sens de celles de Van Dongen et al. (2010), qui n'observent pas d'AD parmi les sept variables étudiées dans leur corpus de 583 fœtus actuels. Une discordance mineure est apparue dans le fait que plusieurs travaux antérieurs identifient une AD au niveau de la longueur maximale de l'humérus (Schultz, 1923 ; Steele et Mays, 1995), résultat qui n'a pas été retrouvé dans le corpus archéologique. Les résultats respectifs de Schultz (1923), identifiant des humérus majoritairement plus longs du côté droit, et Steele et Mays (1995), qui observent pour cet os une AD en faveur du côté gauche, apparaissent toutefois comme contradictoires, et le corpus de Steele et Mays (1995) est par ailleurs très réduit (14 individus). Avec cinq variables significatives dont deux seulement touchant à des longueurs maximales, nous considérons que nos résultats confirment et prolongent ceux des études incluant un protocole d'évaluation à la fois de l'AD et de l'AF comme celle de Van Dongen et al. (2010), à savoir que la latéralisation préférentielle ne semble pas avoir d'expression au niveau osseux au cours de la période périnatale.

\section{Interprétation biologique des résultats sur l'AF}

Les variables identifiées comme exprimant une AF significative étaient, au contraire, nombreuses dans le corpus archéologique et concernaient un grand nombre de variables qui n'étaient pas testées dans les études antérieures (notamment les dimensions des métaphyses). Ces AF présentaient une amplitude située entre 1 et $7 \%$, correspondant à des variations inférieures à $0,5 \mathrm{~mm}$. Ces résultats sont apparus comme cohérents avec les variations attendues et se sont insérés dans la variabilité documentée dans les publications antérieures. Bots et al. (2015) identifient en effet des niveaux d'AF entre 0,6 et $5 \%$, ce qui est observé aussi dans les travaux de Van Valen (1962) (5\%) et de Cuk et al. (2001) (entre 2 et $4 \%$ ).

La pertinence de l'étude des AF en tant qu'estimateur du niveau de stabilité du développement de sujets en période périnatale a été mise en évidence dans la littérature scientifique (Livshits et al., 1988 ; Van Dongen et al., 2009 ; 2010 ; Özener et Graham, 2014), tout comme a pu être souligné l'intérêt de leur analyse en contexte bio-anthropologique (Storm, 2010). La présence d'AF a en premier lieu été montrée comme supérieure parmi les individus porteurs d'anomalies génétiques et d'anomalies du développement (Van Dongen et al., 2010 ; Bots et al., 2015), même si cette association entre AF et anomalies n'apparaît pas comme systématique (Van Dongen et al., 2009). Il s'agit ainsi d'une piste particulièrement intéressante pour la caractérisation de l'état de santé des sujets, dans une perspective paléopathologique et pour l'analyse des pressions environnementales subies par le groupe. De même, à l'échelle inter-populationnelle, l'analyse des AF pourrait permettre de comparer les différences de pressions environnementales entre plusieurs 
groupes issus de contextes chrono-géographiques ou sociaux différents, offrant notamment une nouvelle opportunité d'appréciation des conditions de vie des communautés anciennes.

Ces analyses demeurent toutefois confrontées à plusieurs enjeux, dont en premier lieu le fait qu'elles ne sont réalisables que sur des vestiges osseux extrêmement bien conservés. La question du seuil à partir duquel ces AF peuvent effectivement être considérées comme hors-normes demeure également ouverte. Le développement fœtal ne pouvant pas se dérouler, en pratique, dans des conditions aussi optimales que celles décrites par les approches théoriques, un certain niveau d'AF squelettique doit refléter un développement normal in vivo, niveau qui n'est à l'heure actuelle pas documenté. De même, la question de la significativité et de l'interprétation des écarts inter-populationnels demeure ouverte. Les différences observées signaleraient-elles en effet des disparités d'exposition aux facteurs de stress entre les groupes, ou une hétérogénéité de la réaction face à ces stress, touchant notamment à la variabilité des facultés individuelles d'intégration? Là encore, la question des biais induits par la nature potentiellement pathologique des corpus analysés et de la représentativité des résultats par rapport à ceux qui pourraient être observés dans une population naturelle devra être particulièrement prise en considération (cf. supra, Matériel ; Partiot, 2020).

L'analyse des AD et AF dans des corpus de sujets décédés en période périnatale issus de collections archéologiques apparaît donc comme une perspective particulièrement pertinente pour les analyses ostéobiographiques, et notamment pour l'appréhension des perturbations du développement et des facteurs de stress génétiques ou environnementaux (stress alimentaires, alcoolisation fœtale, consanguinité, etc.) dans les populations du passé. L'enjeu majeur de l'approfondissement de cet axe de recherche réside toutefois dans la constitution d'un référentiel à partir de collections au contexte environnemental et sanitaire connu.

\section{Remerciements}

Les auteurs remercient en premier lieu V. Delattre (Inrap CIF - UMR 6298 ARTeHIS) et l'Inrap pour leur avoir donné accès à la collection de Blandy-les-Tours, ainsi que le Service des Antiquités du Soudan et la Mission de Saï pour l'accès à la collection de la nécropole $8 \mathrm{~B}-51$. CP et FS remercient également plus particulièrement Y. Heuzé (UMR 5199 PACEA), S. Villotte (UMR 5199 PACEA) et V. Sparacello (UMR 5199 PACEA) pour leurs conseils et avis lors de l'élaboration de l'étude, et S. Van Dongen (Université d'Anvers) pour son avis sur les aspects statistiques du protocole. Nous remercions également les deux relecteurs anonymes pour leurs remarques et suggestions. Cette étude a été financée par une bourse de recherche doctorale attribuée au premier auteur par le ministère de l'Enseignement supérieur et de la Recherche, et grâce au soutien pratique et financier du laboratoire d'accueil, l'UMR 5199 PACEA.

\section{Liens d'intérêts}

Les auteurs déclarent ne pas avoir de liens d'intérêts.

\section{Références}

Adalian P (2001) Évaluation multiparamétrique de la croissance fœtale - Applications à la détermination du sexe et de l'âge. Thèse, Université de la Méditerranée, Marseille, France, 372 p

Auerbach BM, Ruff CB (2006) Limb bone bilateral asymmetry: Variability and commonality among modern humans. Journal of Human Evolution 50(2):203-221

Bots J, Ten Broek CMA, Belien JAM et al (2015) Higher limb asymmetry in deceased human fetuses and infants with aneuploidy. Scientific Reports 4(1):3703

Cooke J (2004) Developmental mechanism and evolutionary origin of vertebrate left/right asymmetries. Biological Reviews 79(2):377-407

Cuk T, Leben-Seljak P, Stefancic M (2001) Lateral asymmetry of human long bones. Variability and Evolution 9(1):79-23

Delattre V (2008) Les sépultures de nouveau-nés au Moyen-Âge : l'hypothèse d'un sanctuaire à répit précoce à Blandy-les-Tours (France, Seine-et-Marne). In: Gusi F, Muriel S, Olaria C (eds) Nasciturus, infans, puerulus vobis mater terra: la muerte en la infancia. (Servei D'investigacions Arqueologiques I Prehistoriques), pp 183-210

Duday H, Laubenheimer F, Tillier AM (1995) Sallèles d'Aude Nouveau-nés et nourrissons gallo-romains. Centre de Recherches d'Histoire Ancienne vol. 144 Série Amphores 3, Les Belles Lettres, Paris, $146 \mathrm{p}$

Engmann C, Walega P, Aborigo RA et al (2011) Stillbirths and early neonatal mortality in rural Northern Ghana: Stillbirths and early neonatal mortality. Tropical Medicine \& International Health 17(3):272-282

Fazekas IG, Kósa F (1978) Forensic foetal osteology. Akadémiai Kiadó, Budapest, 344 p

Fishel A (1929) Entwicklung des Menschen. Julius Springer, Berlin, $824 \mathrm{p}$

Gawlikowska A, Czerwiński F, Konstanty-Kurkiewicz V et al (2007) X-ray evaluation of symmetry development of human metatarsal bones in different periods of fetal life. Medical Science Monitor 13(6):BR131-BR135

Guillon M, Portat E, Sellier P (2002) Provins : une fouille programmée (Seine et Marne). Archéopages 6:14-26

Klingenberg CP (2003) A developmental perspective on developmental instability: theory, models and mechanismst. In: Polak M (ed) Developmental instability: causes and consequences. Oxford University Press, Oxford, pp 14-34

Kujanová M, Bigoni L, Velemínská J et al (2008) Limb bones asymmetry and stress in medieval and recent populations of central Europe. International Journal of Osteoarchaeology 18(5):476-491

Lin LI-K (1989) A concordance correlation coefficient to evaluate reproducibility. Biometrics 45:255-268

Livshits G, Davidi L, Kobyliansky E et al (1988) Decreased developmental stability as assessed by fluctuating asymmetry of morphometric traits in preterm infants. American Journal of Medical Genetics - Part A 29(4):793-805 
Livshits G, Kobyliansky E. (1989) Study of genetic variance in the fluctuating asymmetry of anthropometrical traits. Annals of Human Biology 16(2):121-129

Maureille B, Peressinotto D, Murail P et al (2006) La nécropole 8 B51 de l'île de Saï (Province du Nord, Soudan). Archéologie du Nil Moyen 10:181-202

Murail P, Maureille B, Peressinotto D et al (2004) An infant cemetery of the Classic Kerma period (1750-1500 Bc, Island of Saï, Sudan). Antiquity 78(300):267-277

Özener B, Graham JH (2014) Growth and fluctuating asymmetry of human newborns: influence of inbreeding and parental education. American Journal of Physical Anthropology 153(1):45-51

Palmer AR (1994) Fluctuating asymmetry analyses: a primer. In: Markow TA (ed) Developmental instability: its origins and evolutionary implications. Springer Netherlands, Kluwer, Dordrecht, pp 335-364

Palmer AR, Strobeck C (1986) Fluctuating asymmetry: measurement, analysis, patterns. Annual Review of Ecology, Evolution, and Systematics 17(1):391-421

Palmer AR, Strobeck C (1992) Fluctuating asymmetry as a measure of developmental stability: implications of non-normal distributions and power of statistical tests. Acta Zoologica Fennica 191:57-72

Partiot C (2018) Diversité biologique des enfants décédés en période périnatale et traitements funéraires au Kerma classique. Les exemples de la nécropole 8B-51 (Kerma classique, Soudan) et des cimetières de Blandy-les-Tours ( $\mathrm{X}^{\mathrm{e}}-\mathrm{XII}{ }^{\mathrm{e}}$ siècle, France) et de Provins (XIII - XVIII ${ }^{\mathrm{e}}$ siècle, France). Thèse de doctorat, Université de Bordeaux, $736 \mathrm{p}$

Partiot C (2020). Du giron au cercueil : regard bioarchéologique sur la mortalité périnatale. ArkeoGazte: Revista de arqueología-Arkelogia aldizkaria 10:109-126

Partiot C, Guillon M, Peressinotto D et al (2020) Cervical ribs in human early life: morphological variability and first identification as a morbidity criterion in a past population. Journal of Anatomy 237(1):119-132

Portat E (2018) Comportements funéraires autour de la mortalité foto-infantile en France septentrionale (XIII - -XVIII ${ }^{\mathrm{e}}$ siècles). Approche transdisciplinaire. Thèse de doctorat, Université Paris 1 Panthéon-Sorbonne

Schmitt A, Georges P (2008) Quelle démarche suivre pour estimer l'âge au décès à partir du squelette ? In: Charlier $\mathrm{P}(\mathrm{ed})$ Manuel pratique de paleopathologie humaine. De Boccard, Paris, pp 269-280

Schultz AH (1923) Fetal growth in man. American Journal of Physical Anthropology 6(4):389-399

Schutkowski H (1990) Zur Geschlechtsdiagnose von Kinderskeletten: morphognostische, metrische und diskriminanzanalytische Untersuchungen. Georg-August-Universität
Schutkowski H (1993) Sex determination of infant and juvenile skeletons: I. Morphognostic features. American Journal of Physical Anthropology 90(2):199-205

Séguy I (2018) Morts avant que d'être : le paradoxe des fotus et des mort-nés : introduction. In: Charrier P, Clavandier G, Gourdon V et al (eds) Perspectives Historiques. Morts avant de naître : la mort périnatale, Presses universitaires François Rabelais, Tours, pp 23-38

Sellier P, Tillier AM, Bruzek J (1997) À la recherche d'une référence pour l'estimation de l'âge des fœtus, nouveau-nés et nourrissons. Anthropologie et Préhistoire 108:75-87

Steele J, Mays S (1995) Handedness and directional asymmetry in the long bones of the human upper limb. International Journal of Osteoarchaeology 5:39-49

Storm RA (2010) Human skeletal asymmetry. A study of directional and fluctuating asymmetry in assessing health, environmental conditions, and social status in English populations from the $7^{\text {th }}$ to the $19^{\text {th }}$ Centuries. Thèse de doctorat, University of Bradford, $350 \mathrm{p}$

Tang AC, Verstynen T (2002) Early life environment modulates 'handedness' in rats. Behavioural Brain Research 131(1-2):1-7

Van Dongen S (2006) Fluctuating asymmetry and developmental instability in evolutionary biology: past, present and future. Journal of Evolutionary Biology 19(6):1727-1743

Van Dongen S, Gangestad SW (2011) Human fluctuating asymmetry in relation to health and quality: a meta-analysis. Evolution and Human Behavior 32(6):380-398

Van Dongen S, Molenberghs G, Matthysen E (1999) The statistical analysis of fluctuating asymmetry: REML estimation of a mixed regression model. Journal of Evolutionary Biology 12(1):4-102

Van Dongen S, Wijnaendts LCD, Ten Broek CMA et al (2009) Fluctuating asymmetry does not consistently reflect severe developmental disorders in human fetuses. Evolution 63(7): 1832-1844

Van Dongen S, Wijnaendts L, Ten Broek C et al (2010) Human fetuses and limb asymmetry: no evidence for directional asymmetry and support for fluctuating asymmetry as a measure of developmental instability. Animal Biology 60(2):169-182

Van Valen L (1962) A study of fluctuating asymmetry. Evolution 16(2):125-142

Wood JW, Milner GR, Harpending HC et al (1992) The osteological paradox: Problems of inferring prehistoric health from skeletal samples. Current Anthropology 33(4):343-370

Zupan J, Åhman E (2006) Neonatal and perinatal mortality: Country, regional and global estimates. World Health Organization, Genève, $69 \mathrm{p}$ 


\section{ANNEXES}

\section{Annexe 1}

Détails statistiques de l'étape E2-1

Un court résumé est fourni ci-dessous, des détails plus complets peuvent être trouvés dans la publication de Van Dongen et al. (1999).

À l'étape E2-1 du protocole, les deux modèles à effets mixtes suivants sont définis :

- modèle complet : $Y_{i j k}=\mu+\beta+I_{i}+S_{i j}+E_{i j k}$

- modèle réduit : $Y_{i j k}=\mu+\beta+I_{i}+E_{i j k}$

avec $Y_{i j k}=$ observation sur l'individu $i$ pour le côté $j$ et la réplication $k, \mu=$ terme constant du modèle ("fixed intercept"), $\beta$ = effet fixe pour le côté (modélise l'AD), $I_{i}=$ effets aléatoires ("random intercept") iid selon une loi normale centrée, $S_{i j}=$ effets aléatoires iid pour le côté, et $E_{i j k}=$ erreurs aléatoires iid.

Le test de significativité pour la présence d'AD consiste à tester (dans le modèle complet) la nullité de l'effet fixe pour le côté. Le test de significativité pour la présence d'AF consiste en un test de rapport de vraisemblance comparant les vraisemblances (REML) du modèle complet et du modèle réduit.

Symboliquement, en pseudo-code R (pour le package lme4), les modèles seraient de la forme suivante :

- complet $<$ - lmer(Measurement $\sim$ Side $+(1 \mid$ Individual $/$ Side $))$

- reduit $<$ - $\operatorname{lmer}($ Measurement $\sim$ Side $+(1 \mid$ Individual $))$

Le test pour la présence d'AD s'obtient alors par l'instruction car::Anova(complet) ; et le test pour la présence d'AF par l'instruction anova(complet, reduit).

Pour la prise de décision à partir des p-valeurs, le seuil de significativité est fixé à la valeur usuelle de 0,05.

\section{Annexe 2}

Variables exprimant une asymétrie directionnelle statistiquement significative dans le corpus ostéologíque, présentées en ordre croissant

\begin{tabular}{|c|l|c|c|}
\hline Variable & Définition & p-valeur & AD (mm) \\
\hline Ra1 & Longueur maximale de la diaphyse & 0,012 & -2 \\
\hline U15 & $\begin{array}{l}\text { Diamètre sagittal maximal de l'extrémité proximale, } \\
\text { du point le plus antérieur du bec coronoïdien à la face postérieure de l'os }\end{array}$ & $9,16 \times 10-4$ & $-0,1$ \\
\hline U14 & Diamètre maximal au milieu de la diaphyse & $4,58 \times 10-4$ & $-0,1$ \\
\hline Fib7 & Diamètre antéro-postérieur maximal de l'extrémité distale & 0,021 & 0,2 \\
\hline Sc1 & Hauteur de la scapula & 0,027 & 0,3 \\
\hline
\end{tabular}

Avec p-valeur : p-valeur des modèles linéaires mixtes ; $\mathrm{AD}$ : asymétrie directionnelle en millimètres, valeurs négatives : plus grande du côté gauche, valeur positive : plus grande du côté droit. Pour la présentation des variables sur planches anatomiques, se rapporter à Partiot (2018) 


\section{Annexe 3}

Variables exprimant une asymétrie fluctuante statistiquement significative dans le corpus ostéologique, présentées en ordre croissant

\begin{tabular}{|c|c|c|c|c|}
\hline Variable & Définition & p-valeur & AF (\%) & $\mathrm{AF}(\mathrm{mm})$ \\
\hline Hu3 & Diamètre maximal au milieu de la diaphyse & 0,048 & 2,7 & 0,1 \\
\hline Ra10 & Diamètre transversal au milieu de la diaphyse & $5,06 \times 10-6$ & 3,3 & 0,1 \\
\hline $\mathrm{Ra} 6$ & Diamètre transversal de l'extrémité proximale & $4,85 \times 10-4$ & 3,7 & 0,2 \\
\hline $\mathrm{Hu} 8$ & Diamètre sagittal de l'extrémité distale & 0,004 & 3,8 & 0,2 \\
\hline Sc8 & Largeur maximale de la cavité glénoïdale & $1,33 \times 10-7$ & 3,8 & 0,2 \\
\hline U13 & Diamètre minimal au milieu de la diaphyse & 0,03 & 6,9 & 0,2 \\
\hline Hu5 & Diamètre maximal de l'extrémité proximale & $1,8 \times 10-4$ & 1,6 & 0,2 \\
\hline Ra11 & Diamètre transversal de l'extrémité distale & $1,33 \times 10-3$ & 1,9 & 0,2 \\
\hline Is2 & Diamètre sagittal maximal de l'ischium & 0,033 & 1,9 & 0,2 \\
\hline Hu6 & Diamètre minimal de l'extrémité proximale & $1,77 \times 10-3$ & 2,1 & 0,2 \\
\hline U110 & Diamètre maximal de l'extrémité distale & $1,09 \times 10-5$ & 4,8 & 0,3 \\
\hline Sc6 & Hauteur de la région glénoïdale & $7,17 \times 10-3$ & 2,3 & 0,3 \\
\hline $\mathrm{I} 18$ & $\begin{array}{l}\text { Diamètre transversal de la région acétabulaire perpendiculaire au } \\
\text { diamètre sagittal maximal de la région acétabulaire, mesure oblique } \\
\text { de haut en bas et de l'extérieur vers l'intérieur }\end{array}$ & $4,65 \times 10-3$ & 3,4 & 0,4 \\
\hline Ti9 & Diamètre transversal maximal de l'extrémité distale & $5,55 \times 10-3$ & 4,2 & 0,4 \\
\hline $\mathrm{Cl} 6$ & Diamètre vertical de l'extrémité médiale & $8,4 \times 10-4$ & 4,8 & 0,4 \\
\hline U16 & $\begin{array}{l}\text { Diamètre transversal perpendiculaire au diamètre sagittal maximal } \\
\text { de l'extrémité proximale, pris du point le plus antérieur du bec } \\
\text { coronoïdien à la face postérieure de l'os }\end{array}$ & $4,42 \times 10-3$ & 6 & 0,4 \\
\hline $\mathrm{Hu} 7$ & Largeur transversale maximale de l'extrémité distale & $2,22 \times 10-6$ & 2,2 & 0,4 \\
\hline Pu1 & Longueur du pubis prise parallèlement au bord supérieur & $2,01 \times 10-4$ & 2,3 & 0,4 \\
\hline Hu1 & Longueur maximale de la diaphyse & $8,29 \times 10-9$ & 0,7 & 0,4 \\
\hline $\mathrm{Il} 1$ & $\begin{array}{l}\text { Hauteur de l'ilium prise du point le plus bas de la région acétabu- } \\
\text { laire au point de la crête iliaque qui en est le plus éloigné. Ce point } \\
\text { est situé au voisinage du milieu de la crête iliaque }\end{array}$ & $7,41 \times 10-5$ & 1,3 & 0,4 \\
\hline Ti7 & Diamètre transversal maximal de l'extrémité proximale & $1,23 \times 10-6$ & 3,3 & 0,5 \\
\hline Sc3 & $\begin{array}{l}\text { Largeur prise entre l'extrémité caudale de la cavité glénoïdale et } \\
\text { le point du bord médial qui est le plus éloigné du centre du versant } \\
\text { vertical de la région glénoïdo-coracoïdienne, dans la zone corres- } \\
\text { pondant à la naissance de l'épine }\end{array}$ & $1,28 \times 10-3$ & 1,7 & 0,5 \\
\hline Ti1 & Longueur de la diaphyse & $3,20 \times 10-11$ & 0,7 & 0,5 \\
\hline $\mathrm{Cl} 3$ & $\begin{array}{l}\text { Largeur externe maximale prise entre les points les plus éloignés } \\
\text { des bords antérieur et postérieur de l'extrémité latérale }\end{array}$ & $1,80 \times 10-12$ & 5,9 & 0,5 \\
\hline $\mathrm{I} 12$ & $\begin{array}{l}\text { Hauteur de l'ilium prise du point situé à l'extrémité inférieure de } \\
\text { la linea arcuata au point de la crête iliaque qui en est le plus éloigné }\end{array}$ & $6,87 \times 10-3$ & 1,9 & 0,6 \\
\hline $\mathrm{I} 13$ & $\begin{array}{l}\text { Largeur de l'ilium de l'épine iliaque antéro-supérieure } \\
\text { à l'épine iliaque postérosupérieure }\end{array}$ & $4,70 \times 10-14$ & 1,9 & 0,6 \\
\hline $\mathrm{I} 14$ & Diamètre antéro-postérieur maximal de la surface auriculaire & 0,029 & 4,9 & 0,6 \\
\hline $\mathrm{C} 11$ & Longueur maximale & $4,27 \times 10-15$ & 1,7 & 0,8 \\
\hline
\end{tabular}

Avec p-valeur : p-valeur des modèles linéaires mixtes ; AF : asymétrie fluctuante ; $\%$ : par rapport à la variable ; (mm) : quantité d'AF en millimètres. Pour la présentation des variables sur planches anatomiques, se rapporter à Partiot 2018 